\title{
Utility of Usg-Guided Fine Needle Aspiration Cytology in The Diagnosis of Intra-Abdominal Masses
}

\author{
Jinu Abraham Glaxon ${ }^{1 *}$, Prema Saldanha ${ }^{2}$ and Sheela Vasudevan ${ }^{3}$ \\ ${ }^{1}$ Department of pathology, Sree Gokulam Medical College And Research Foundation, Thiruvananthapuram, India \\ ${ }^{2}$ Department of pathology, Yenepoya Medical College, Mangalore, India \\ ${ }^{3}$ Department of pathology, Sree Gokulam Medical College And Research Foundation, Thiruvananthapuram
}

\begin{abstract}
Background: Fine needle aspiration cytology (FNAC) with the aid of imaging modalities like Ultrasonography (USG) and Computed Tomography (CT) is an increasingly used technique in diagnosing intra-abdominal masses, both non neoplastic and neoplastic. This study was undertaken to evaluate the utility of image guided cytology in various palpable and non-palpable intra-abdominal lesions.

Materials and Methods: A retrospective study was conducted in the Department of Pathology over a period of two years to find the utility of USG guided FNAC in intra-abdominal masses. Percutaneous ultrasound guided FNAC was conducted in a total of 66 cases with palpable and non-palpable intra-abdominal lesions were included in the study.

Results: A total of 66 patients with intra-abdominal lesions were included in the study. The age of the patients ranged from 14 to 90 years. Of these, 48 cases $(72.72 \%)$ were males and 19 cases $(28.78 \%)$ were females. Out of the total aspirates, 7 cases $(10.61 \%)$ were categorised as non-neoplastic, 55 cases $(83.33 \%)$ as neoplastic and 4 cases $(6.06 \%)$ were categorised as inadequate. Thirty one cases (46.96\%) were in hepatobiliary region, 9 cases $(13.63 \%)$ in pancreatic-ampullary region, 8 cases $(12.12 \%)$ in the retroperitoneal lymph nodes, 6 cases $(9.09 \%)$ in the right iliac fossa, 4 cases $(6.06 \%)$ in the gastrointestinal tract, 3 cases $(4.54 \%)$ of ovarian masses and one case $(1.51 \%)$ of renal mass.

Conclusion: FNAC is a simple, safe, quick, non invasive and inexpensive procedure. USG guided assisted FNAC plays an important role in the diagnosis and differentiation of neoplastic and non neoplastic intra-abdominal lesions.
\end{abstract}

Keywords: Fine Needle Aspiration, Intra-Abdominal Masses, Cytology

\section{Introduction}

The evaluation and diagnosis of deep intra-abdominal and intra-pelvic lesions is a fairly difficult procedure. These lesions can present as palpable or as deep nonpalpable masses. These lesions can be benign, malignant, or inflammatory and distinction between them is vital for patient management. Imaging techniques may not always distinguish between benign and malignant lesions. Many inflammatory conditions such as tuberculosis and liver abscesses can be misleading to the radiologists. ${ }^{[1]}$

FNAC is a well established, simple, rapid and cost effective diagnostic technique with high sensitivity and specificity and low complication rates. ${ }^{[1]}$ Radiologically assisted cytology namely USG-guided and computed tomographyguided fine needle aspiration (FNA) is an effective way to obtain diagnostic material.

Interaction and teamwork between the cytopathologist and radiologist is important so as to obtain maximum diagnostic yield and increased overall accuracy.

In case of malignant lesions, FNA cytology has shown $100 \%$ specificity and is considered to be the first choice for evaluation of abdominal lesions. ${ }^{[2]}$ Accurate diagnosis of malignancy, the cell type and pattern will be beneficial for patients in determining the therapies and thereby improving prognosis.

The present study aims to evaluate the utility and diagnostic value of image guided cytology in various palpable and non-palpable intra-abdominal lesions.

\section{Material and Methods}

This is a retrospective study in which a total of 66 patients with intra-abdominal mass lesions are included. FNAC of the intra-abdominal lesions is carried out at the Department of Pathology over a period of two years.

The relevant findings, clinical history, physical examination and radiological findings were obtained from the records in the pathology department.

The patients are first subjected to an ultrasonographic evaluation in order to assess the origin of the lesion and its relationship with the adjacent organs. After taking aseptic precautions, a percutaneous FNAC of the lesion is done under real-time USG guidance. 
A 20ml disposable plastic syringe and a 22 gauge needle is used in case of palpable lesions. For deep-seated lesions, a 20-22 gauge spinal needle of $9 \mathrm{~cm}$ length is used. A transabdominal approach, by using the most direct route is made and care is taken to obtain material from the most solid portion of the lesion.

After insertion of the needle, strong suction is applied and the needle tip is moved in and out by about $5 \mathrm{~mm}$. The needle is then withdrawn and smears prepared.

Air dried smears are stained with Giemsa and wet fixed smears are fixed in a Koplin's jar containing 95\% alcohol and stained using Papanicolaou and H\&E stains, respectively. Special stains are used wherever required.

The smears are then classified as benign, malignant, and inadequate or non representative.

Table 1: Cytological diagnosis of the lesions

\begin{tabular}{|c|c|c|c|c|c|}
\hline Site & Cytological diagnosis & Males & Females & $\begin{array}{l}\text { Total number } \\
\text { of cases }\end{array}$ & $\begin{array}{c}\text { Percentage of } \\
\text { cases }(\%)\end{array}$ \\
\hline \multirow[t]{5}{*}{ Liver } & & 25 & 6 & 31 & 46.97 \\
\hline & Primary & 15 & 3 & & \\
\hline & Secondary & 10 & 3 & & \\
\hline & known primary & 5 & 2 & & \\
\hline & unknown primary & 5 & 1 & & \\
\hline \multirow[t]{3}{*}{ Pancreas } & & 7 & 2 & 9 & 13.64 \\
\hline & Neoplasms & 5 & 1 & & \\
\hline & Nonneoplastic lesions & 2 & 1 & & \\
\hline $\begin{array}{l}\text { Retro-peritoneal } \\
\text { lymph nodes }\end{array}$ & NonHodgkin's lymphoma & 6 & 2 & 8 & 12.12 \\
\hline \multirow[t]{5}{*}{ Right iliac fossa } & & 3 & 3 & 6 & 9.09 \\
\hline & Nonneoplastic lesions & 2 & 1 & & \\
\hline & Tuberculosis & 2 & 2 & & \\
\hline & Metastasis & 1 & 0 & & \\
\hline & Recurrent ca colon & 0 & 1 & & \\
\hline \multirow[t]{3}{*}{ GIT } & & 3 & 0 & 3 & 4.55 \\
\hline & Ca stomach & 2 & 0 & & \\
\hline & Ca colon & 1 & 0 & & \\
\hline Ovary & Carcinoma & 0 & 3 & 3 & 4.55 \\
\hline Gall bladder & Ca gallbladder & 0 & 1 & 1 & 1.51 \\
\hline Kidney & Transitional cell Carcinoma & 1 & 0 & 1 & 1.51 \\
\hline Inadequate & & 3 & 1 & 4 & 6.06 \\
\hline TOTAL CASES & & 48 & 18 & 66 & 100 \\
\hline
\end{tabular}

\section{Results}

Sixty six cases of intra-abdominal lesions were included in the study.

The age of the patients ranged from 14 years to 90 years.

Majority of the patients were male comprising of 48 cases $(72.72 \%)$ followed by 19 cases $(28.78 \%)$ females. Out of the total aspirates, maximum number 31 cases $(46.97 \%)$ were hepatic lesions. The cytological diagnosis of the different lesions along with the site and sex distribution of each is given in Table 1.

According to cytomorphology, majority of the cases were classified as neoplastic constituting 55 cases $(83.33 \%)$, followed by 7 cases $(10.61 \%)$ of non-neoplastic, and 4 cases $(6.06 \%)$ were categorised as inadequate or nonrepresentative. 


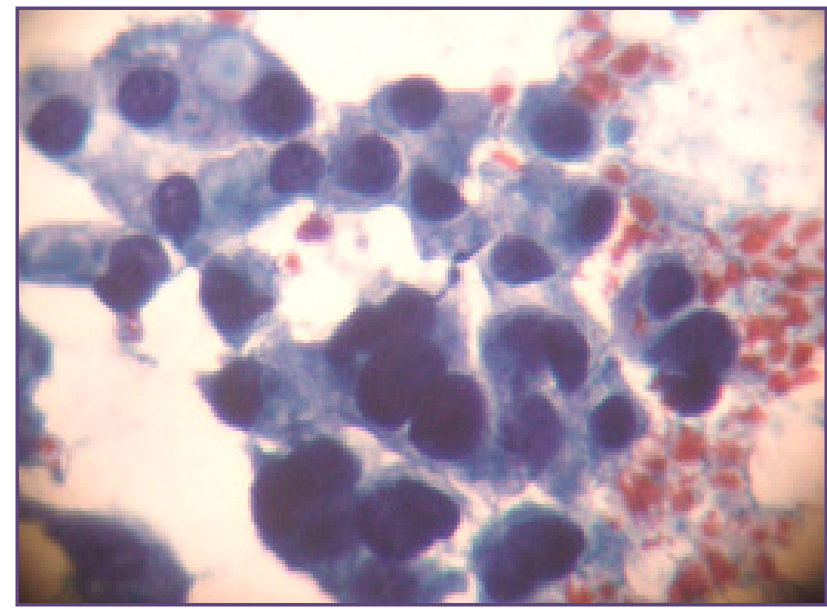

Fig. 1: Primary hepatocellular carcinoma composed of polygonal cells with severe anisokaryosis seen arranged in a sinusoidal pattern (Papanicolaou 40x).

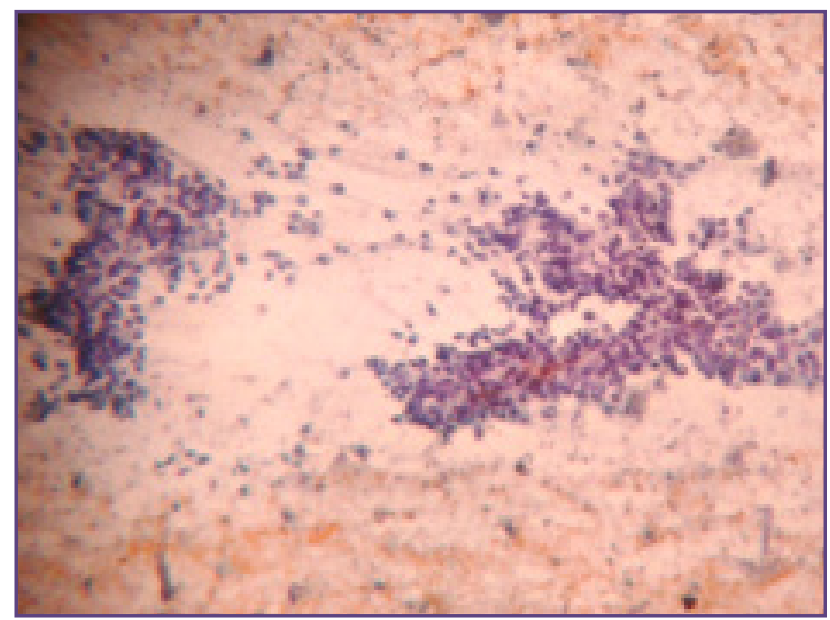

Fig. 3: Carcinoma pancreas showing cells arranged in a branching papillaroid pattern and showing nuclear atypia and crowding (Papanicolaou 40x).

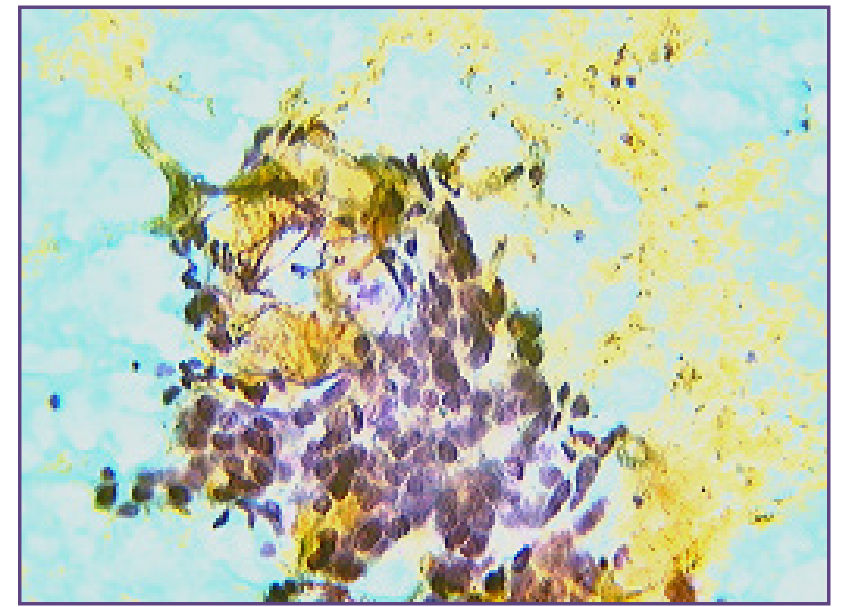

Fig. 2: Metastasis to liver showing malignant epithelial cells in an acinar pattern (Papanicolaou 40x).

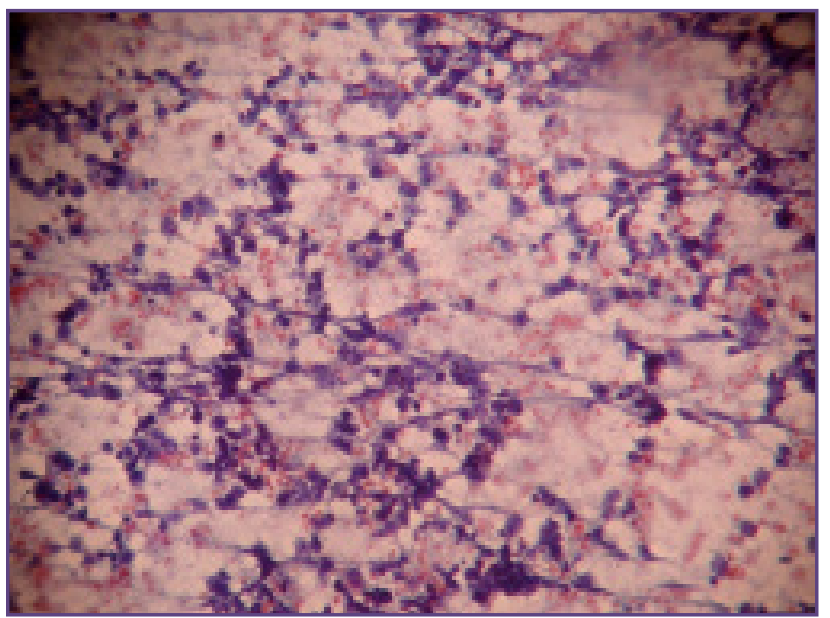

Fig. 4: Non Hodgkin Lymphoma showing monotonous population of atpical lymphoid cells (Giemsa 40x).

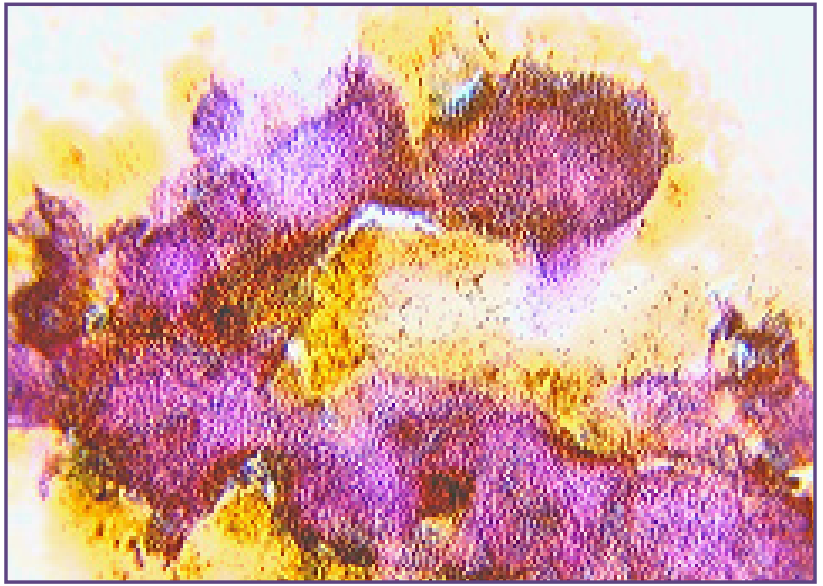

Fig. 5: Transitional cell carcinoma kidney showing highly cellular smears with multilayered papillary structures and tumor cells having pleomorphic nuclei with coarse chromatin (Papanicolaou 40x). 


\section{Discussion}

Radiologically assisted FNA cytology of palpable and nonpalpable intra-abdominal lesions offers the advantage of rapid and accurate diagnosis with a minimum of surgical intervention. Fine needle aspiration cytology is simple, safe, repeatable and superior in terms of cost effectiveness and rapidity of diagnosis. ${ }^{[1]}$ The main advantage of FNAC in intra-abdominal mass lesions is that it helps to determine the nature of the lesion, non-neoplastic or neoplastic and whether benign or malignant. Further, the diagnostic accuracy of FNAC of deep seated lesions dramatically increases when interpreted by an experienced cytopathologist having good communication with the radiologists and clinicians.

In the present study, $4(6.06 \%)$ cases out of a total of 66 cases were inadequate, hence the diagnostic yield was $93.9 \%$. This is higher and comparable to other studies. ${ }^{[4]}$, [5], [6], [7]

Of the total of 66 cases, it was observed that a majority of cases, $83.33 \%$ were reported to be malignant and remaining cases $10.60 \%$ were benign and non- neoplastic lesions. These results were similar and comparable to other studies. ${ }^{[4],[5],[6],[10]}$

Liver was the most common site for FNAC in this study comprising of $31(46.96 \%)$ cases. This is comparable to other similar studies. ${ }^{[4], ~[5], ~[6], ~[9], ~[11] ~ A m o n g ~ t h e s e, ~ a l l ~ o f ~ t h e ~}$ cases were confirmed to be malignant of which $18(58.1 \%)$ cases were reported to be primary hepatocellular carcinoma composed of polygonal cells with severe anisokaryosis seen arranged in a sinusoidal pattern (Figure 1). Thirteen $(41.9 \%)$ cases were metastasis to liver showing malignant epithelial cells in an acinar pattern (Figure 2). This is in contrast to literature ${ }^{[1],[3]}$ and other studies ${ }^{[4],[5],[6],[1]]}$ where metastatic tumors of liver is the most common hepatic malignancy encountered.

There were nine cases of pancreas among which majority $6(9.1 \%)$ cases were pancreatic carcinoma with cells arranged in a branching papillaroid pattern and showing nuclear atypia and crowding. (Figure 3).

Among the eight cases of retroperitoneal lymphnodes, all were diagnosed to be Non Hodgkins lymphoma showing a monotonous population of atypical lymphoid cells (Figure 4). This finding is in contrast with other studies ${ }^{[4]}$, ${ }^{[12]}$ where majority of the lesions were found to be reactive or inflammatory.

Six cases of lesions in the right iliac fossa were studied in which majority $(6.06 \%)$ of cases were tuberculosis showing epithelioid cell clusters in a background of caseous necrotic material followed by one case each of metastases and recurrent carcinoma colon. This finding is in contrast to a large series study ${ }^{[6]}$ where majority of the right iliac fossa masses were malignant lesions.

In various studies ${ }^{[4]},{ }^{[11]}$ FNAC was done in more number of cases of ovarian carcinomas, while in the present study FNAC was done in only three $(4.54 \%)$ cases of ovarian carcinomas.

In our study, two cases of carcinoma stomach and one case each of carcinoma colon and carcinoma gall bladder was observed.

One case of transitional cell carcinoma of the kidney was reported showing highly cellular smears with multilayerd papillary structures and tumor cells having pleomorphic nuclei with coarse chromatin. (Figure 5)

\section{Conclusion}

USG-GUIDED FNAC is a simple, safe, quick, inexpensive and highly accurate diagnostic procedure. It plays an important role in aiding the diagnosis of palpable and non-palpable intra-abdominal masses, as well as in differentiating between non-neoplastic and neoplastic lesions. High sensitivity and specificity in diagnosing the malignant lesions helps in planning appropriate surgical interventions. A good co-operation between clinician, radiologist and pathologist is of utmost imporatnce to improve the diagnostic accuracy.

\section{References}

1. Orell S.R., Sterret G.F., Whitaker D. : Fine needle aspiration cytology. 5th Edition Elsevier Churchill Livingstone, 2011.

2. Pitman MB. Fine needle aspiration biopsy of the liver. Principal diagnostic challenges. Clin Lab Med 1998; 18: 483- 506.

3. Juan Rosai, VJ. Desmet, GO. Nelson. Rosai and Ackerman's Surgical Pathology. 9th ed. 2005; Mosby.

4. Dosi S, Gupta G, Kawatra M, Chakrabarti P, Agrawal P, Jain M. Role of radiological-assisted cytology in intraabdominal lesions: A 3 years experience in a tertiary care center. International Journal of Applied and Basic Medical Research. 2016;6:101-105.

5. Reyaz TA, Summyia F, Isma N, Nazia B, Adil S, Sameena K, Humaira B, Naila N, Ambreen B. USG guided fine needle aspiration cytology of intra-abdominal and pelvic masses in Kashmir: A study at tertiary care hospital. International Journal of Medical research and health sciences.2016 Jan 1;5:169-75.

6. Naiding M, Goswami A, Singh S. Role Of Usg Guided Fnac In Diagnosis Of Intra-Abdominal And Intrapelvic Masses. International Journal of Advanced Research. 2017;5:222-33. 
7. Nautiyal S, Mishra RK, Sharma SP. Routine and ultrasound guided FNAC of intra-abdominal lumps - A comparative study. J Cytol. 2004;2:129-32.

8. Zawar M.P., Dr. Bolde S., Dr. Shete S.S. Correlative study of fine needle aspiration cytology and histology in intraabdominal lumps. SMJ 2007; 4-8.

9. Khan AA, Jan GM, Wani NA. Fine needle aspiration of intra-abdominal masses for cytodiagnosis. J Indian Med Assoc. 1996;94:167-8.
10. Ahmad SS, Akhtar K, Akhtar S, Abrari A, Nasir A, Khalid $\mathrm{M}$, et al. Ultrasound guided fine needle aspiration biopsy of abdominal masses. JK Sci. 2006;8:200-4.

11. Adhikari RC, Tuladhar A, Shrestha S, Sharma SK. Deepseated thoracic and abdominal lesions: Usefulness of ultrasound guided fine needle aspiration cytology, a 3 year experience. Nepal Med Coll J. 2010;12:20-5.

12. Porter B, Karp W, Forsberg L. Percutaneous cytodiagnosis of abdominal masses by ultrasound guided fine needle aspiration biopsy. Acta Radiol Diagn (Stockh) 1981;22:663-8.

*Corresponding author:

Dr Jinu Abraham Glaxon, Assistant Professor, Department of pathology, Sree Gokulam Medical College And Research Foundation, Venjaramoodu,

Thiruvananthapuram, India Pin - 695607

Phone: +919544800523

Email: jinu232@gmail.com

Financial or other Competing Interests: None. 